Departamento de Ciência e Tecnologia, Secretaria de Ciência e Tecnologia e Insumos Estratégicos do Ministério da Saúde

Correspondência | Correspondence: Decit Departamento de Ciência e Tecnologia do Ministério da Saúde

Esplanada dos Ministérios

Bloco G sala 845

70058-900 Brasília, DF, Brasil

\section{Participação do Brasil no IV Annual Meeting of Health Technology Assessment International em Barcelona,} Espanha - 2007

\section{Brazil participation on the IV Annual Meeting of Health Technology Assessment International in Barcelona Spain - 2007}

Nos últimos anos, o crescente aparecimento de inovações tecnológicas em saúde tem atuado como instrumento de pressão sobre os sistemas de saúde que gerenciam recursos limitados (Nunes \& Rego, 2002). Se por um lado, tecnologias são incorporadas sem a garantia de eficácia e efetividade, de outro, existem tecnologias obsoletas que continuam a ser utilizadas nos serviços, mesmo não representando mais a melhor opção terapêutica ou diagnóstica.

Por isso, os gestores de saúde necessitam de informações coerentes sobre os benefícios, os riscos e os custos das tecnologias e seu impacto sobre os serviços de saúde.

Nesse contexto, a avaliação de tecnologias em saúde (ATS) apóia os gestores em saúde na tomada de decisão em relação à incorporação ou abandono das tecnologias. Constitui-se processo abrangente, por meio do qual são avaliados os impactos clínico, social e econômico da utilização das tecnologias, emergentes ou já existentes, desde a pesquisa e desenvolvimento até a obsolescência. Este processo leva em consideração aspectos como eficácia, segurança, efetividade, custo-efetividade, entre outros (Goodman, 1998; Hunink e Glasziou, 2001).

Definem-se como tecnologias em saúde medicamentos, equipamentos, procedimentos técnicos, sistemas organizacionais, educacionais e de suporte, programas e protocolos assistenciais, por meio dos quais a atenção e os cuidados com a saúde são prestados à população (BRASIL, 2005).

O Departamento de Ciência e Tecnologia da Secretaria de Ciência, Tecnologia e Insumos Estratégicos do
Ministério da Saúde (Decit/SCTIE/MS) é a instância governamental no Ministério da Saúde designada para implementar, monitorar e difundir a ATS no Sistema Único de Saúde (SUS). O Decit é responsável por identificar as demandas por estudos em ATS advindas das áreas técnicas do Ministério da Saúde, de secretarias estaduais e municipais. Além disso, analisa e define o tipo mais adequado de avaliação a ser realizada, elabora os pareceres técnicos científicos ou viabiliza a contratação da instituição de pesquisa com expertise comprovada para a realização de estudos complexos.

A atuação do Ministério da Saúde em ATS se destaca com a elaboração da Política Nacional de Gestão de Tecnologias em Saúde (PNGTS), que é o instrumento norteador para os atores envolvidos na gestão dos processos de avaliação, incorporação, difusão, gerenciamento da utilização e retirada de tecnologias no sistema de saúde.

Outro importante avanço foi o fato de o Brasil passar a integrar, por intermédio do Decit, a International Network of Agencies for Health Technology Assessment (INAHTA - Rede Internacional de Agências de Avaliação de Tecnologias em Saúde), desde maio de 2006. A INAHTA foi fundada em 1993 e agrega cerca de 50 agências de 22 países, com o objetivo de cooperar com a difusão de informações e estudos na área de ATS. Com sede na Suécia, a INAHTA possui entre seus membros agências das Américas Latina e do Norte, Europa, Austrália e Nova Zelândia.

Visando a estimular a troca de experiências na área e congregar conhecimentos para colaborar na estrutura- 
ção do processo da ATS no Brasil, o Decit participou do IV Annual Meeting of Health Technology Assessment International (Encontro anual de Avaliação de Tecnologias em Saúde), em Barcelona.

O presente artigo descreve os principais resultados do referido evento, das visitas oficiais a algumas agências de ATS na Espanha e a síntese da $15^{\circ}$ reunião de membros da INAHTA.

\section{Annual Meeting of Health Technology Assessment International}

O evento aconteceu de 17 a 20 de junho de 2007, no Palácio de Congressos de Barcelona, Espanha, cujo tema foi ATS para a saúde pública baseada em evidências, do qual participaram cerca de mil representantes de diversos países.

O Ministério da Saúde foi representado pelo Decit por meio de uma comitiva constituída da diretora do Departamento, a coordenadora de Avaliação de Tecnologias em Saúde e quatro técnicos da área, que apresentaram quatro pôsteres intitulados: Brazilian investment in professional qualification in Health Technology Assessment; Brazilian Policy of Health Technology Management: opportunities and challenges to Technology Assessment in health system; Priority setting for health technology assessment in Brazilian Public Health System; Assessment of surgical treatments for people suffering from morbid obesity: the Brazil's case.

Também participaram do encontro outros 16 representantes das agências nacionais de vigilância sanitária e saúde suplementar de São Paulo, Minas Gerais, Rio de Janeiro, Rio Grande do Norte, Rio Grande do Sul e Distrito Federal.

No evento foram apresentados 573 resumos, e o Brasil esteve em quarto lugar entre os países com trabalhos aprovados. Representantes do Brasil, do Programa de Engenharia Biomédica da Universidade Federal do Rio de Janeiro, tiveram pôster premiado, com trabalho intitulado Planning cancer care based on population needs: a simulation model for public health management in Brazil.

Durante o evento, articulou-se cooperação técnica e troca de experiências com diversas agências que tradicionalmente atuam com ATS, bem como com aquelas nos países em desenvolvimento, que estão iniciando suas atividades nesta prática.

No dia 17 de junho, aconteceram pré-conferências com foco em temáticas atuais como:

- Introdução a programas efetivos de rastreamento nos serviços de Saúde Pública - cujo objetivo era ajudar os participantes a discutirem ferramentas para avaliação da qualidade dos programas de rastreamento nos Sistemas de Saúde de seus países.

- Introdução à Avaliação de Tecnologias em Saúde - apresentado por Clifford Goodman, um dos iniciadores da discussão sobre ATS no mundo.

- Como implementar os resultados da avaliação de uma tecnologia - discutiu as barreiras da implementação dos resultados de uma avaliação e contou com o relato das experiências do National Institute for Clinical Excellence and Health (NICE), da Inglaterra.

- Ultrapassando as fronteiras da gestão da informação em ATS - contou com a presença de diversos atores envolvidos com a disseminação das informações advindas do processo de ATS instalado em vários países, como Canadá, Inglaterra e Estados Unidos.

- EUnetHTA: desenvolvimento de metodologias transnacionais - teve por objetivo apresentar os resultados do desenvolvimento de métodos para a prática da ATS pela Rede Européia de Avaliação de Tecnologias em Saúde.

- Envolvimento de pacientes e cidadãos em ATS - mostrou a importância da participação desses atores no processo de ATS.

Ainda no período pré-congresso, houve simpósios que contaram com a participação de empresas da área médica, e discutiram sobre vacina contra o vírus papiloma humano, tecnologias emergentes e tele-medicina.

Nos três dias do encontro, entre 18 e 20 de junho, houve grandes plenárias, compostas por temas relevantes mundialmente. Os debates destacaram a busca por recursos para avaliação de tecnologias em saúde pública, a avaliação das estratégias adotadas para pandemia de influenza e a implantação de ATS nos países em desenvolvimento. O último debate contou com a presença de um representante do Brasil, que destacou os desafios para a implementação das decisões baseadas em evidência em saúde pública.

Adicionalmente, ocorreram sessões orais relativas às grandes áreas temáticas, como:

- Obesidade - um dos grandes temas do encontro, discutiu a avaliação de intervenções para o controle da obesidade em escolares e adultos, entre elas: drogas, dietas, programas de prevenção e promoção da saúde e cirurgia bariátrica.

- Métodos em ATS - abordagem de aspectos metodológicos para a prática da ATS, como avaliação econômica, adoção de aspectos éticos e socioculturais e utilização de revisões rápidas para orientar decisões. 
- Pacientes e cidadãos - discussão sobre ferramentas para a disseminação de informações para os pacientes e a sociedade civil em geral, visando a garantir o envolvimento destes atores no processo de ATS; debateu-se o principio da precaução aplicado para saúde pública e para a prática clínica.

- Tecnologias emergentes - tele-medicina, drogas para doenças órfãs, rastreamento e terapêutica em oftalmologia (glaucoma, retinopatia diabética, catarata) e câncer de mama, intervenções em saúde mental, entre outras.

- ATS para a prática clínica e para orientar decisões políticas - abordagem da aplicabilidade das evidências científicas para diversas condições de saúde, como diabetes, Aids, diarréia e doenças cardiovasculares; avaliação do uso de diretrizes clínicas nos serviços de saúde, impacto e as dimensões de contexto que influenciam na utilização dos informes de ATS nas decisões.

- Capacitação - avaliação de experiências como o Programa Ulysses, mestrado internacional em gerenciamento e avaliação de tecnologias em saúde, da Universidade de Montreal, e o Programa Executive Training for Research Application (EXTRA), da Canadian Health Services Research Foundation.

- ATS, indústrias e sistemas de saúde - importante discussão sobre a participação das indústrias no processo de produção do conhecimento em ATS e a racionalização destes conhecimentos quando aplicados à saúde pública.

- Avaliação de intervenções em saúde pública - discussão das estratégias de rastreamento de doenças, como câncer colorretal e de mama; abordou o uso das evidências científicas para implantação de programas anti-tabagismo, anti-alcolismo e de redução da poluição do ar; discutiu rastreamento e vacinação contra o vírus papiloma humano, profilaxia para doenças virais como influenza e rotavírus.

Dentre esses temas discutidos, foi observada consonância com as seguintes atividades do Decit:

\section{Métodos em avaliação de tecnologias em saúde}

- Definição de prioridades por meio de critérios definidos e oficinas que envolvem representantes de comunidade científicas e gestores, como o Grupo de Trabalho Permanente em Avaliação de Tecnologias em Saúde do Conselho de Ciência, Tecnologia e Inovação em Saúde do Ministério da Saúde (GT $\mathrm{ATS} / \mathrm{CCTI} / \mathrm{MS})$.

- Desenvolvimento e validação de Diretrizes Metodológicas para Estudos em ATS, que orientam como produzir revisões rápidas, intituladas Pareceres Técnico-Científicos.

\section{Avaliação de tecnologias em saúde, indústrias e sistemas}

- Implantação da Política Nacional de Gestão de Tecnologias em Saúde define diretrizes para gestão, incorporação, monitorização e retirada de tecnologias do sistema de saúde. A criação de fórum para análise e decisão sobre as incorporações tecnológicas no sistema de saúde.

\section{Capacitação}

- Promoção de cursos para formação de cerca de 170 técnicos dos serviços de saúde municipais, estaduais, agências reguladoras e ministério da saúde em avaliação e gestão de tecnologias em saúde (especialização e mestrado).

- Estruturação da Rede Brasileira de Avaliação de Tecnologias em Saúde (Rebrats), a partir da junção das instituições de ensino e pesquisa e os técnicos e gestores que estão participando do processo de formação. Integração com a Rede Nacional de Pesquisa Clínica em Hospitais de Ensino.

\section{Informação, conhecimento e disseminação em avaliação de tecnologias em saúde}

- Estruturação de área temática dentro do Portal Saúde(www.saude.gov.br $\rightarrow$ ciência e tecnologia $\rightarrow$ ATS), para divulgar os resultados dos estudos de ATS promovidos pelo Decit e facilitar acesso às principais bases de dados internacionais, como a da INAHTA. A base do INAHTA fornece resumos de estudos completos de ATS, revisões sistemáticas e avaliações econômicas realizadas pelas agências do mundo.

\section{Projetos em avaliação de tecnologias em saúde:}

- Promoção de revisões sistemáticas e estudos de custo efetividade relativos aos programas do SUS.

- A partir da observação dos Projetos induzidos nas Agências Internacionais de ATS, identificou-se harmonia entre as prioridades correntes de pesquisa do Decit. Intervenções para a obesidade e diabetes são avaliadas considerando as especificidades de cada país. Cabe destacar ainda que temas negligenciados, como a farmacoterapia da leishmaniose, serão priorizados respeitando as características epidemiológicas do Brasil.

\section{Visitas oficiais às agências de avaliação de tecnologias em saúde na Espanha}

Os representantes do Decit fizeram visitas a algumas das agências de avaliação de tecnologias em saúde da Espanha: 
- Agencia de Evaluación de Tecnologías Sanitaria do Instituto de Salud Carlos III (AETS) - Madrid.

- Agencia de Evaluación de Tecnologías Sanitarias de Andalucía (AETSA) - Sevilla.

- Agència d'Avaluació de Tecnologia i Recerca Mediques, atualmente Agência para Qualidade, Pesquisa e Avaliação em Saúde (AQuRAHealth) - Barcelona.

O objetivo das visitas foi conhecer a estrutura e funcionamento das referidas agências. Ressaltam-se os seguintes pontos:

- As agências foram instituídas há cerca de 14 anos e estão vinculadas às autoridades sanitárias das comunidades autônomas da Espanha;

- Assessoram a tomada de decisão dos gestores públicos e possuem canais formais para comunicação com sociedades de profissionais e de pacientes;

- Atuam com equipe fixa e com consultores externos, na elaboração de metodologias de ATS, na capacitação e na produção de informes;

- Produzem os informes de ATS e também contratam estudos por meio de convocatória pública. Os estudos são direcionados a tomada de decisão que inclui: compilação e análise de bases de dados, revisões sistemáticas e estudos primários com recomendações específicas *Possuem legislação própria e definem prioridades publicadas anualmente. Os temas são definidos pelas áreas técnicas das autoridades sanitárias das comunidades autônomas, do Ministério da Saúde e de representantes de sociedades profissionais e de comunidade científica. No âmbito regional, as prioridades são identificadas nos serviços primários e hospitalares. As prioridades são reavaliadas a cada dois anos.Elaboram fichas técnicas sobre tecnologias emergentes e adotam a estratégia de uso tutelado para aquelas de alto custo que necessitam de avaliação de efetividade nos serviços tendo por base um protocolo clínico.

- Recentemente, criaram uma plataforma eletrônica de gestão do conhecimento com objetivo de promover o intercâmbio das atividades das agências de ATS das comunidades autônomas.

\section{$15^{\circ}$ encontro anual da International Network of Agencies for Health Technology Assessment}

Ao fim do IV Annual Meeting of Health Technology Assessment International, aconteceu o $15^{\circ}$ Encontro anual da INAHTA, em que os dirigentes do Brasil (Decit) também estavam presentes. Os temas discutidos permearam áreas como: parcerias externas, desenvolvimento de websites, impacto da ATS, qualidade das revisões rápidas, educação e treinamento, ética em ATS.

Em conclusão, a participação no IV Annual Meeting of Health Technology Assessment International, a visita às agências de ATS e o encontro entre os membros da INAHTA, foram oportunos tanto para capacitação da equipe do Decit, como para identificar experiências aplicáveis no Brasil.

De modo geral, a área de Avaliação de Tecnologias em Saúde do Ministério da Saúde segue as principais diretrizes mundiais para o campo. Também, temas como a vacina contra o vírus papiloma humano, intervenções para obesidade, drogas para doenças órfãs, papel da indústria, entre outros, foram discutidos no evento e estão presentes na realidade brasileira.

A inserção brasileira no contexto internacional da avaliação de tecnologias em saúde é parte do compromisso do Departamento de Ciência e Tecnologia, na estruturação e disseminação do campo no Sistema Único de Saúde. 